SANDIA REPORT

SAND97-0964 • UC-405

Unlimited Release

Printed April 1997

\section{RECEIVED \\ JUN 301997 \\ OSTI}

\title{
Three Dimensional Visualization to Support Command and Control
}

Gerald A. Van Slambrook

Prepared by

Sandia National Laboratories

Albuquerque, New Mexico 87185 and Livermore, California 94550

Sandia is a multiprogram laboratory operated by Sandia

Corporation, a Lockheed Martin Company, for the United States

Department of Energy under Contract DE-AC04-94AL85000.

Approved for public release; distribution is thiminted

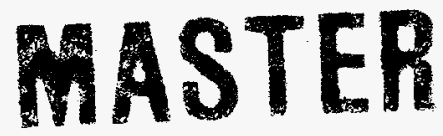

\section{An Sandia National Laboratories}

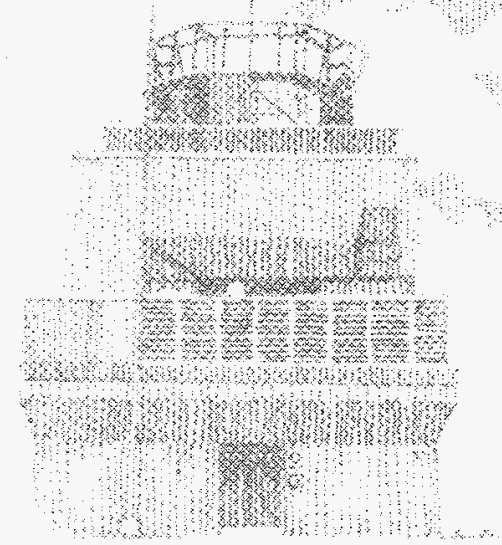

SF 29000 (8-81)

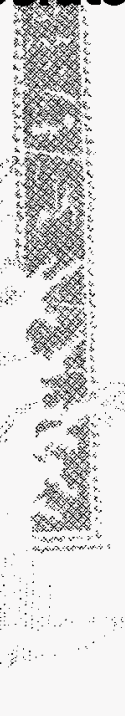

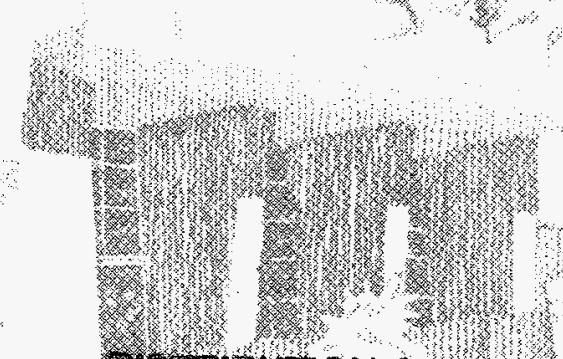

11

IS OSTRIBUTON OF THS DOCUMENT IS UNLIMTTED , w 


\section{DISCLAIMER}

This report was prepared as an account of work sponsored by an agency of the United States Government. Neither the United States Government nor any agency thereof, nor any of their employees, make any warranty, express or implied, or assumes any legal liability or responsibility for the accuracy, completeness, or usefulness of any information, apparatus, product, or process disclosed, or represents that its use would not infringe privately owned rights. Reference herein to any specific commercial product, process, or service by trade name, trademark, manufacturer, or otherwise does not necessarily constitute or imply its endorsement, recommendation, or favoring by the United States Government or any agency thereof. The views and opinions of authors expressed herein do not necessarily state or reflect those of the United States Government or any agency thereof. 


\section{ACKNOWLEDGEMENTS}

Dan Holloway for his dedication and technical excellence in modeling and building the satellite simulation, terrain visualization, and multi-dimensional data visualization systems. Sharon Stansfield for her collaborative efforts in building the Virtual Reality Research Laboratory and for her work with MIT graduate student Craig Cooke on the robotics research project. 


\section{TABLE OF CONTENTS}

Section

1.0 INTRODUCTION

2.0 A VR Model

3.0 The VR Research System

4.0 Satellite Application

5.0 Robotics Application

6.0 Conclusions

7.0 References
Page

1

3

9

11

16

17

18

A-1 
iv

LIST OF FIGURES

Figure

Page

1 The Human/Real World Interface Types

3

2 The VR Simulation Model

5

3 GPS Satellite Crosslink \& Downlink Communications

12

4 GPS Satellite Orbit \& Path Simulation

12

5

Dynamic Sensor Coverage Map

13

6

Terrain Elevation Data

14

7

Multi-dimensional Data Visualization

15 


\section{LIST OF TABLES}

Table

Page

1

VR Research Laboratory Hardware

9

2

VR Research Laboratory Software

10 


\section{INTRODUCTION}

\subsection{Executive Summary}

This report documents the results of a two year LDRD project concerning the exploration and demonstration of the utility of virtual reality ${ }^{1}$ (VR) in robotics and satellite command and control applications. The robotics work centered around a system to build accurate graphical models of physical environments, some of which are too dangerous for human access. The application centered around the command and control of robots used by DOE for environmental restoration tasks. The objective was to allow the user to interactively build a model of a remote environment by superimposing stereo graphics onto live stereo video. The user marked points on objects in the video and the computer calculated the dimensions and pose of the object. Using this information, a model of the object was added to the graphical world. Other modes, such as voice and sound, were included to aide the user in building the objects in the virtual environment.

The satellite work addressed the fusion of multiple data sets or models into one synergistic display for more effective training, design, and command and control of satellite systems. It also explored alternative worlds for displaying terrain and abstract data sets. The objective was for an operator to view data in a more natural and intuitive way by being immersed within the satellite's environment and from this perspective quickly and effectively convert the fused information to knowledge and actions.

To accomplish this work, it was necessary to survey the existing virtual reality technology including hardware and software systems and to build a research laboratory utilizing as many commercially available components as possible upon which the applications and research could be built. This report includes a summary of the fundamental elements of this laboratory.

It was necessary to develop a model for the various virtual reality applications. That model and its applicability to a number of applications is also discussed in this report.

\subsection{Concept}

Virtual reality is a new paradigm for the human-machine interface. It revises both the way we think about computers and the way we think with them. Virtual images presented directly to the senses provide a participant with a total global experience. The participant is surrounded by computer-synthesized information -- a spatial world in which the person and the computer can more effectively communicate. The user interacts with this inclusive medium by looking at objects, pointing his or her hands and giving verbal commands. The medium permits virtual objects which appear to be real to be touched and manipulated by the participant. Virtual worlds may be shared even over long distance. Virtual interfaces solve many existing interface problems and empower new and novel interfaces for teleoperation, computer-aided design, education, medical imaging, command and control, and much more. With such broad applicability, it is easy to see why virtual

1. The use of the term virtual reality encompasses the entire field of teleoperator and virtual environment systems. The popular terms for such systems includes but is not limited to cyberspace, virtual environments, teleoperation, telerobotics, augmented reality, and synthetic environments. In the text of this document, virtual reality is used as a generic term to describe any such system and may be abbreviated as VR. 
reality has captured the attention and imagination of not only scientists but even the most casual of computer users. With systematic development, virtual interfaces can be one of the greatest advances of our age and a boon to industry.

Virtual reality represents a new division of labor between the human and the computer. A partnership in which the computer does what it does best -- high speed calculations, redundant operations, data storage and retrieval and humans do what they do best -- reasoning, creating, overall context, and correlation of information to form conclusions. The VR system's primary task becomes one of creating a complete environment for the participant based upon a set of information in the form of a model. We interact with this virtual environment through our senses, reasoning our way through some situations, reacting immediately to others just as we do in the real world. The computer responds to our actions and even our voice, intuitively and intelligently, constantly changing the environment in response to our actions or commands.

In any virtual reality system, the basic elements are a human being as an operator or participant, a computer or set of computers, and one or more devices linking the human to the computer(s). The computers are programmed to generate a synthetic environment that can be interacted with by the operator. The distinction between a teleoperator system and virtual reality system is in purpose the teleoperator system aids the user in sensing and interacting with the real world. A virtual reality system is intended to alter reality for the operator by generating a completely synthetic environment. An augmented reality system is a combination of the two - a real world representation enhanced with computer generated information.

Two primary concepts exist in virtual reality - immersion and interactivity. Immersion is the sense that we have "entered" a computer generated world and that we are "there". Interactivity means that we can interact with that world in ways that are meaningful or intuitive. Without these two elements, VR looses most of its advantage and utility over traditional graphical systems. 


\section{A VR Model}

The diagram in Figure 1. illustrates the differences between the "normal" human experience of interacting with the real world and that of interacting in a telerobotic system and a virtual environment. The VR model discussion to follow builds upon this fundamental construct.

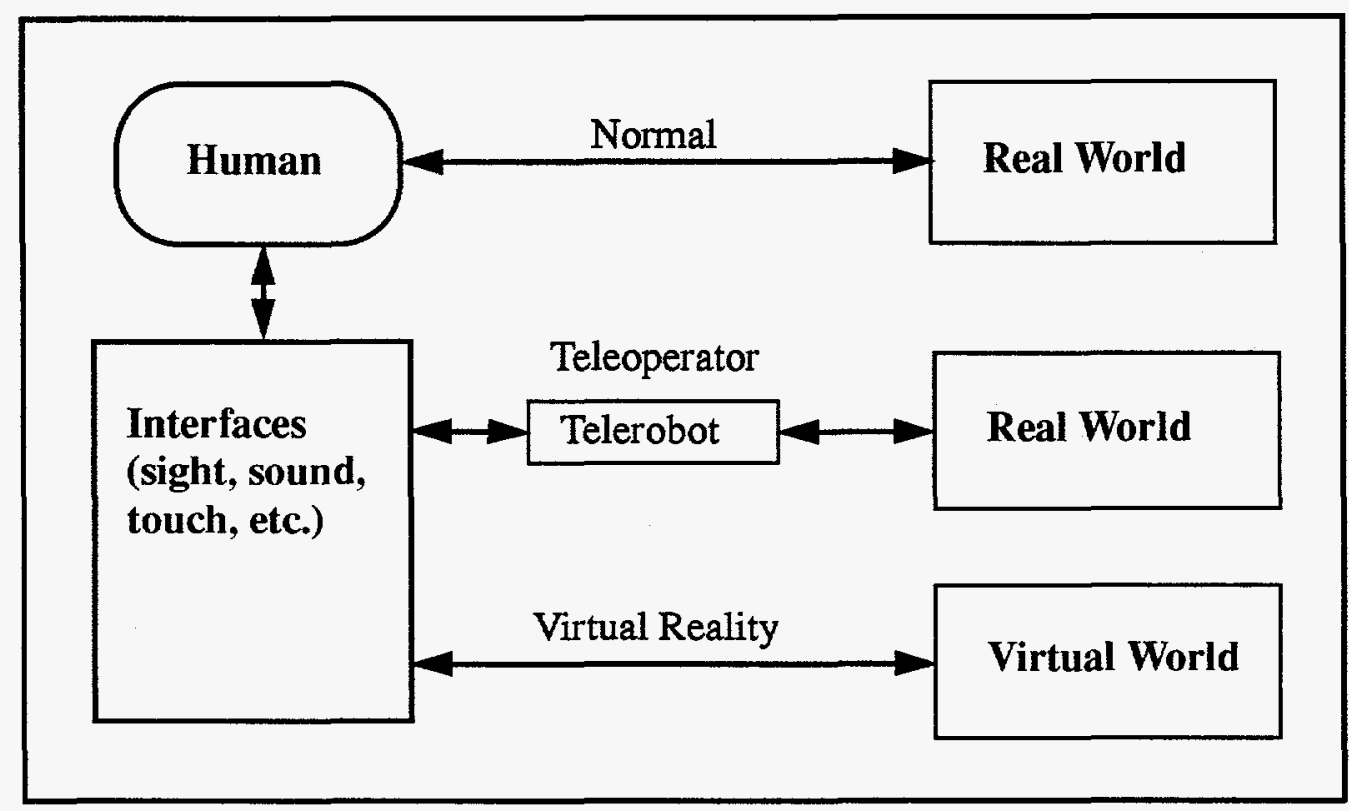

Figure 1. The Human/Real World Interface Types.

\subsection{Concept}

Structured programming and software engineering methodologies are in common use today and have done much to improve the "art of programming". The classic waterfall model of software development shown below:

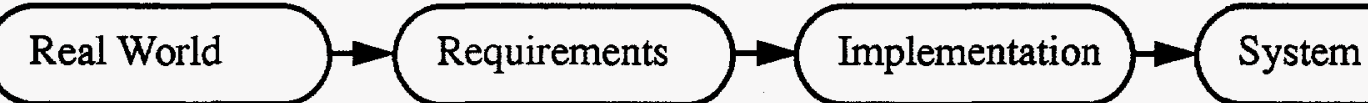

This is an iterative process which is being continually refined and improved in a number of methodologies and available CASE tools. One refinement of this software development model is the "essential software process" where the flow is described as:

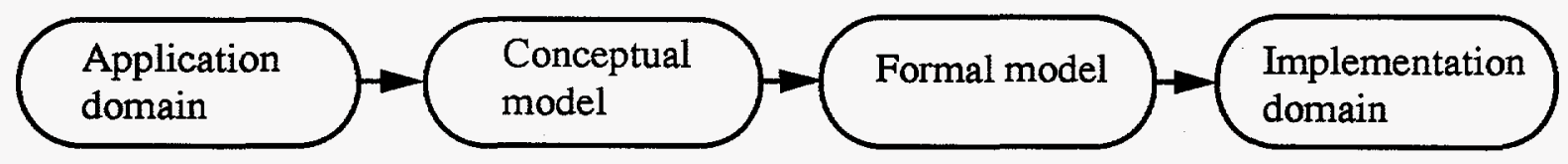

In either case the process is a transformation from a need to a software product that meets that need. There is, in one form or another, two models, one built in terms of the application domain 
and another built in terms of the implementation domain. There has typically been a large semantic gap between the two models due, primarily, to the lack of correlation between the real world and the system. That is, the results and interface to the developed system is "disconnected" from the real world. This is not to say that the results are incorrect, only that it is much more difficult to measure their correctness. Virtual reality, in essence, is an implementation model that reduces that gap by making it possible to produce results in terms of the real world. Validation of the result is almost immediate because it can be in a form that is familiar to the application expert whose needs it is the system is trying to meet.

The concept we are presenting here is an approach to virtual reality applications that ties the application domain models more closely to the implementation models. It discusses VR applications primarily in terms of the models and the interactions with those models for a particular application.

\subsection{VR Development Process Guidelines}

The development process involves modeling in three different phases while a model is being refined. These include model building, model application, and model adaptation. The process passes through each of these phases starting with building the application followed by adaptation. Thereafter, there may be transitions between any two phases with no particular ordering. Of particular importance in this process is the ability to quickly adapt to modifications required to realistically model the application. This requirement makes rapid prototyping tools nearly a necessity.

The model building phase considers the form and size of raw data to be input to the model, whether from real world sources (such as a VR camera) or the abstract world (such as seismic data). The type of model is also of importance. A static model presents an unchanging appearance to the viewer, such as a background image, while a dynamic model changes appearance or behavior with changes in input data. In addition, whether the viewer interacts with the model or whether the model is generated off-line and then displayed to the viewer is important. Often a model must be generated as a series of scenes with off-line processing and then played back to animate data in time or perhaps change perspectives with changing movements and actions of the observer. This requires storage of a database of scenes with rapid access a necessity for real-time viewing. Specifiying model components using object-oriented techniques lends itself to this process. One may not only define the characteristics and attributes of an object, but also the state machines that define its behavior and the methods by which one may interact with it.

The model application phase makes actual use of the models built. The object state machines describing behavior are implemented and the appearance of the model is viewed in a Virtual Reality display. In addition, sensors and effectors are experienced for the environment modeled. Here the model is examined in light of its intended use. A control program, the simulation manager directs the model execution and directs outputs to the observer.

The model adaptation phase is a stage of examining the desirability of the model and validating its closeness to the desired characteristics. Relationships are modified and enhancements proposed before returning to another phase. 


\subsection{Fundamentals of VR}

The discussion of VR applications requires at least a fundamental understanding of the elements and issues involved with a VR system. Therefore, Figure 2. depicts the basic components of a system and their interactions. Comments are added to the connecting arrows to highlight the devices or issues involved with that connection. A discussion of each element of the diagram follows.

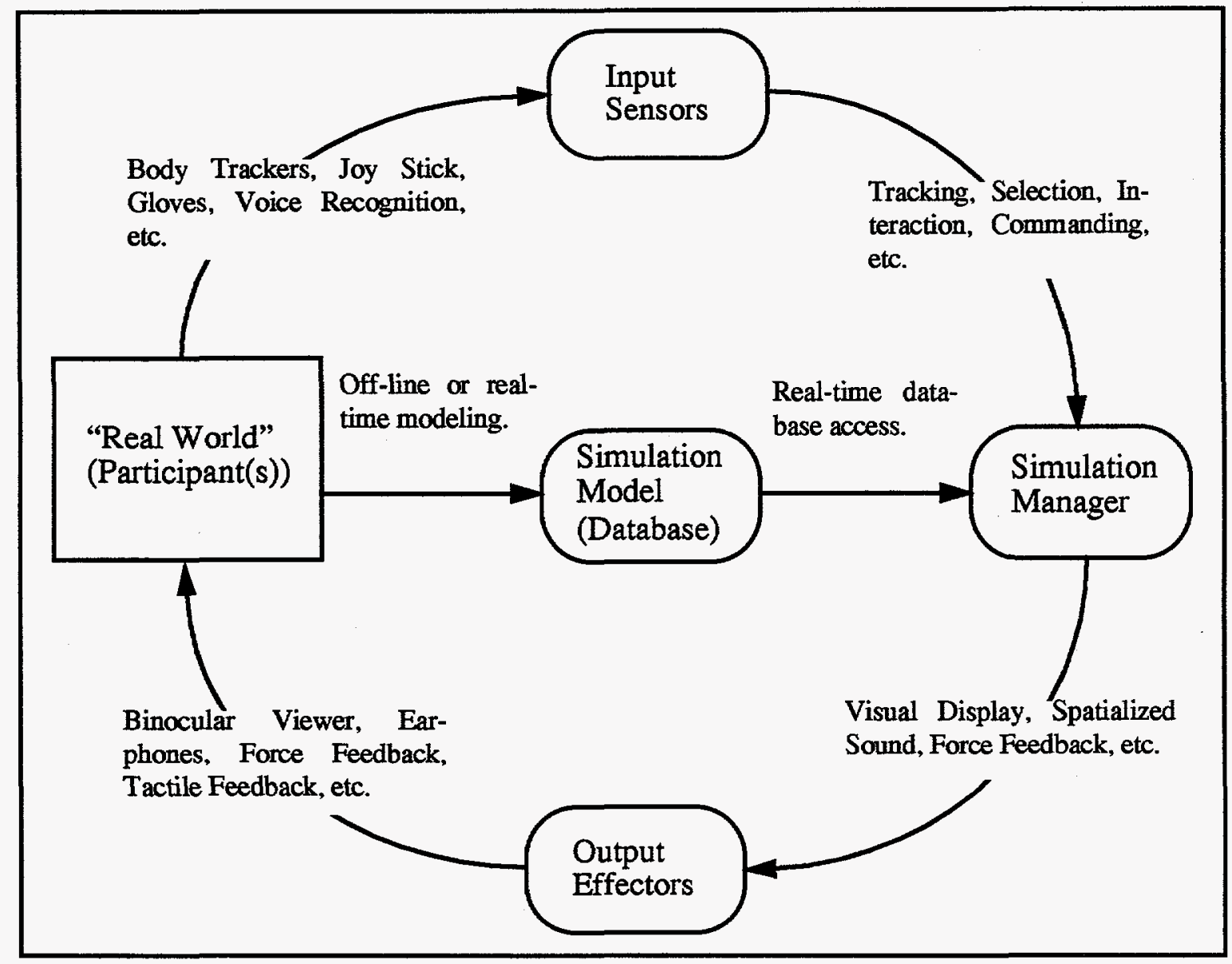

Figure 2. The VR Simulation Model

\subsubsection{Simulation Manager}

This is the core component of the computer side of the human-machine interface whereas the human is represented by the "participant" block on the opposite side of the diagram. The simulation manager contains the computer(s), graphics engine(s), interface components, and software that together perform the following three basic steps:

1. Read the input sensors.

2. Correlate the input sensors to the simulation model and update the model based upon these inputs as well as dynamic models such as mechanical, physical, and animation. 
3. Produce the virtual display based upon $1 \& 2$. In the context of VR and for the purposes of this report, "virtual display" means not just a stereo graphics image, but any and all implemented sensory feedback systems such as sound, touch, and force.

Two primary issues come into play in the simulation manager, frame update rate and sensor lag. Frame update rate is simply how many display updates can be produced per second. The generally accepted minimum rate is 12 frames per second with an optimal goal of 30. Lag is the perceived delay between activation of an input sensor and the resultant effect in the VR display. This phenomenon is device dependent and is a result of the amount of time it takes for the hardware to detect a change and communicate that change to the system. In the case of the tracking system, the effect would be that the participant moves but does not see the image change until some later instance.

\subsubsection{Input Sensors}

This component provides the human-to-machine inputs necessary to the simulation manager for updates to the simulation model and ultimately the production of the VR display. The first and most fundamental input sensor is the body tracker. This may include multiple sensors which are capable of transmitting six degrees of information to the system comprised of location (xyz) and orientation (roll, pitch, and yaw). Before a completely immersive experience can be generated, the simulation manager needs to know where the participant is looking, therefore, the first part of the body which must be tracked is the head. With this information, the simulation manager can produce a visual display of the environment with the correct perspective for the viewer. A second very common body tracker is for the hand. With this information, the simulation manager can detect and respond to the participants hand position for the purpose of interaction with objects existing within the virtual environment. Finally, a full body tracking system may be used to create a representation of the individual in the environment viewable by other participants in what is referred to as a shared virtual world.

Examples of other input sensors related directly to human interaction are voice recognition, gloves that sense the angles of the joints, 6D mice, and joysticks all of which are intended as natural and intuitive interfaces between the user and the environment.

Finally, other inputs from the "real world" outside of the participant may be real-time data sources from other systems such as satellite sensors, state-of-health, or remote sensors on a robot. These inputs could be read and displayed directly by the simulation manager or used to dynamically update the simulation model.

\subsubsection{Output Effectors}

The output effectors provide the feedback to the participant (machine-to-human interface) and encompass all of the devices that are intended to give the impression of being in another world. First and foremost of these is the visual display which is stereo for purposes of depth information and wide in field of view for a sense of immersion. Other dimensions are added with devices which can produce effects such as spatialized sound, force feedback, and a sense of touch. 
The primary issues with output effectors are the details of the display and the human factors. Detail of the display has to do primarily with resolution and the accuracy of the actions and representations of the objects in the environment. The human factors are very complex and have to do with how we perceive the world around us and how virtual worlds are created that interface correctly and meaningfully to the human mind. Another human factor issue is how much realism is required to produce the desired effect in a VR application. Much research and experimentation are still required to answer these questions, however, the technology is far enough advanced that it can be beneficial in a number of applications. Complex scenes still result in slow frame update rates and appear somewhat cartoon-like, however, the concept of "suspended disbelief" wherein our minds bridge the gap between the real world and the simulated world provide enough realism to show the benefits of the technology.

\subsubsection{Real World and Participants}

This, of course, is the human side of the system and everything that exists outside of the virtual environment. The human factors of a virtual environment were addressed briefly in the section on output effectors. The other factors to be aware of here are that the participant is affected by the physical surroundings at the same time he or she is experiencing the virtual environment. Depending upon the application, it may or may not be important to minimize the "real world" inputs. In the case of telerobotics, on the other hand, it is desirable to have the real world represented in the virtual world by means of such things as stereo video inputs and sensor status overlays. The arrow connecting this component to the simulation model covers this case as well as where it is desired to create the simulation model from real world inputs. This will be addressed further in the section on modeling.

\subsubsection{Simulation Model}

The simulation model or database is, in essence, the description of the virtual environment that is to be produced in the VR display by the simulation manager. The model may include static and dynamic information according to the particular application

\subsubsection{Static Models}

A static model defines those objects that are fixed in the virtual environment and cannot be changed or affected by the dynamic model or input sensors. Some examples of static models would be:

- A star database that produces a realistic background display in a satellite demonstration.

- A fixed data set being viewed but not changed in the virtual environment such as SAR data from a site being analyzed in an intelligence application.

- A training model which is used to evaluate a users performance in a given scenario.

- A building or structure such as produced in a CAD system. 


\subsubsection{Dynamic Models}

A dynamic model defines the objects that are affected by input sensors and other stimuli that may be internal models themselves with properties such as physics, temporal relationships, animation sequences, and mechanics. It contains the state machine for the VR simulation and receives updates from the "real world" or input sensors and provides responses to those inputs. The dynamic model is the key ingredient to producing an interactive virtual environment dictating the rules that are in effect for a given application. For example, the participant reaches out, touches a ball, and the ball rolls according to the pressure applied and its physical properties of size and weight.

\subsubsection{Modeling Issues}

For most applications today, the simulation model exists in resident memory and thus is accessed at rates high enough to maintain a desirable frame update rate. As models become larger and more complex, it may be necessary to have very high speed access to some form of mass storage devices or other machines which may be performing parallel computations and accesses to a local database.

The more immediate and difficult problem is the one of building the model itself. This may be accomplished in a number of different manners, all of which are today labor intensive.

- The application code may be written to contain the model within itself.

- Objects may be imported from a number of different CAD packages.

- Some VR software packages exist which provide some of the static and dynamic modeling capabilities as well as aids for the implementation of the simulation manager.

- A number of modeling packages exist that fulfill some but not all of the needs of a VR dynamic model.

The point here is that the existing technology makes VR model building difficult and time consuming. The models can be built and applications may be done but more work is needed in this area to ease the process. 


\subsection{The VR Research System}

In order for this research to be successful, it was necessary to build a state-of-the-art laboratory consisting of hardware and software components that could provide a quality high-performance VR capability. Some of these components were upgrades from previous years models as newer and higher performance models became available. Only the final configuration of the laboratory systems are summarized here.

Table 1: VR Research Lab Hardware

\begin{tabular}{|c|c|c|}
\hline Vendor & Product & Description \\
\hline SGI & Onyx & $\begin{array}{l}\text { Reality Engine. Dual pipe-line graphics engine with } \\
256 \mathrm{M} \text { memory, } 4 \text { ea. R } 4000 \text { processors, } 9 \mathrm{~GB} \text { disk, and } \\
\text { Video Lab. }\end{array}$ \\
\hline SGI & Personal IRIS & Graphics development workstation. \\
\hline $\begin{array}{l}\text { FakeSpace } \\
\text { Labs }\end{array}$ & $\begin{array}{l}\text { Black \& White } \\
\text { Boom }\end{array}$ & $\begin{array}{l}\text { Stereo video display and tracking system. Currently } \\
\text { scheduled for upgrade to a two color Boom. }\end{array}$ \\
\hline $\begin{array}{l}\text { FakeSpace } \\
\text { Labs }\end{array}$ & Color Boom & $\begin{array}{l}\text { Two color high resolution video display and tracking } \\
\text { system. }\end{array}$ \\
\hline $\begin{array}{l}\text { FakeSpace } \\
\text { Labs }\end{array}$ & Molly & Slaved stereo video camera system. \\
\hline $\begin{array}{l}\text { Paradigm } \\
\text { Simulations }\end{array}$ & Sound hardware & $\begin{array}{l}\text { Six channel digital sound synthesizer, preamp, and four } \\
\text { speakers. }\end{array}$ \\
\hline $\begin{array}{l}\text { Ascension } \\
\text { Technology }\end{array}$ & Flock of Birds & Two receivers with Extended Range Tracker. \\
\hline $\begin{array}{l}\text { Shooting } \\
\text { Star Tech. }\end{array}$ & ADL-1 & 6D tracker \\
\hline SGI & Crystal Eyes & Stereo glasses for monitor. \\
\hline $\begin{array}{l}\text { Electra } \\
\text { Sonic }\end{array}$ & Microphone & $\begin{array}{l}\text { Cordless microphone for use with the voice recognition } \\
\text { system. }\end{array}$ \\
\hline BG Systems & Flybox & Joy stick with multiple buttons and sliders. \\
\hline Panasonic & Recorder & Video cassette recorder. \\
\hline Sony & Recorder & Laser videodisc recorder \\
\hline Chromatek & Converter & Video scan converter \\
\hline
\end{tabular}


Table 2: VR Research Lab Software

\begin{tabular}{|c|c|c|}
\hline Vendor & Product & Description \\
\hline SGI & IRIX & Unix operating system. \\
\hline SGI & CaseVision & Software development tool. \\
\hline SGI & Explorer & Data visualization. \\
\hline $\begin{array}{l}\text { FakeSpace } \\
\text { Labs }\end{array}$ & $\begin{array}{l}\text { Boom } \\
\text { Library }\end{array}$ & $\begin{array}{l}\text { Boom VR support library for both the } \\
\text { black and white and two color Booms. }\end{array}$ \\
\hline Sense8 & WTK & VR application development toolkit. \\
\hline $\begin{array}{l}\text { Deneb } \\
\text { Robotics }\end{array}$ & IGRIP & $\begin{array}{l}\text { Robotics modeling package modified to } \\
\text { support FakeSpace Boom. }\end{array}$ \\
\hline Silma & CimStation & Robotics modeling package. \\
\hline $\begin{array}{l}\text { Paradigm } \\
\text { Simulations }\end{array}$ & $\begin{array}{l}\text { Audio } \\
\text { Works }\end{array}$ & Sound library \\
\hline $\begin{array}{l}\text { Dragon Sys- } \\
\text { tems }\end{array}$ & Dragon Lab & $\begin{array}{l}\text { Voice command input software (PC ver- } \\
\text { sion). }\end{array}$ \\
\hline $\begin{array}{l}\text { Dynamic } \\
\text { Graphics }\end{array}$ & No name & $\begin{array}{l}\text { Interactive 3D surface generation pack- } \\
\text { age. }\end{array}$ \\
\hline
\end{tabular}




\subsection{Satellite Applications}

Satellite orbit dynamics, sensor coverage planning, state-of-health monitoring, and robotic device control are but a few of the problems facing a satellite ground station operator. Even with current technology, the operator is inundated with data usually in a number of separate and counter intuitive displays. It is difficult at best for an operator to envision exactly what is happening to a satellite under his control. Virtual reality affords a unique opportunity to fuse multiple data sources into one synergistic and interactive display. The operator may be immersed in the satellite environment and in a natural and intuitive manner see, feel, and hear what the satellite sees, feels, and hears. A system like this has potential for simulation and training as will as actual real-time control. The following is a description of just such a system modeled around the concepts described in section 2.

The first effort in this work was to create an earth-centered celestial sphere environment into which satellite models and data could be imported. An important objective was to create enough realism to give a participant the sense that they were suspended in outerspace. The model created included the earth, sun, moon, and a thousand of the brightest stars all in their correct positions in an earth-centered coordinate system. One form of the earth model is a very realistic view as created from actual satellite photographs texture mapped unto a set of polygons approximating a sphere. Another earth model may be viewed in wire-frame depicting lines of latitude and longitude with rough outlines of the continents. This form of the earth model is useful for projecting satellite data onto the earth model where geographical location information may be important.

The next step was to create the ability to simulate various satellites, their orbits, and dynamics. A number of different satellites, including the space shuttle, have been simulated in this environment. Extensive work was conducted on simulating the GPS satellite constellation. This work involved the merging of multiple data sets into one dynamic interactive model in the environment described above. The simulation centers on nuclear detection payloads on-board these satellites and several dynamics related to these systems. Orbit dynamics and their effects on crosslink and downlink communication may be observed and analyzed in this system including simulations of detected events. Figure 3. shows one frame of the simulation. Downlink paths are shown in green, crosslink communications in pink and the satellites themselves in yellow. Time may be controlled in the simulation such that a particular date may be chosen for observation as well as increased or decreased to aid in analysis. Figure 4 . shows one frame of the simulation where the orbit paths and planes are displayed.

Data from a bolide event that was detected by the sensor systems onboard the GPS satellites was reproduced in this simulation for purposes of comparison to ground based human sightings of the event. The model correctly identified the eight satellite sensor systems that actually detected the event. The participant is able to "fly" in the virtual environment to the point of the ground based sighting and view the event as it should have been seen according to the satellite data.

Finally, this simulation was used to dynamically create satellite sensor coverage maps. It is often of interest to be able to describe the region of the earth that is viewable by a specific sensor at a specified time from a spacecraft. If the sensor is a full-earth staring sensor and is not effected by atmosphere or other obstructions, the region of visibility is simply the physical geometrical hori- 


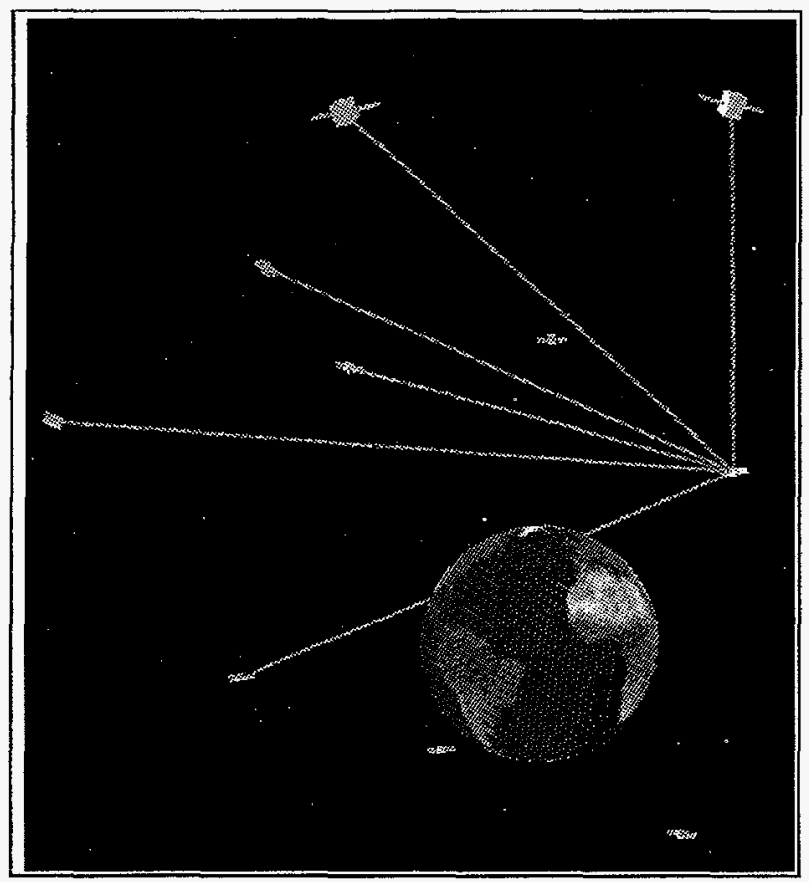

Figure 3. GPS Satellite Crosslink \& Downlink Communications

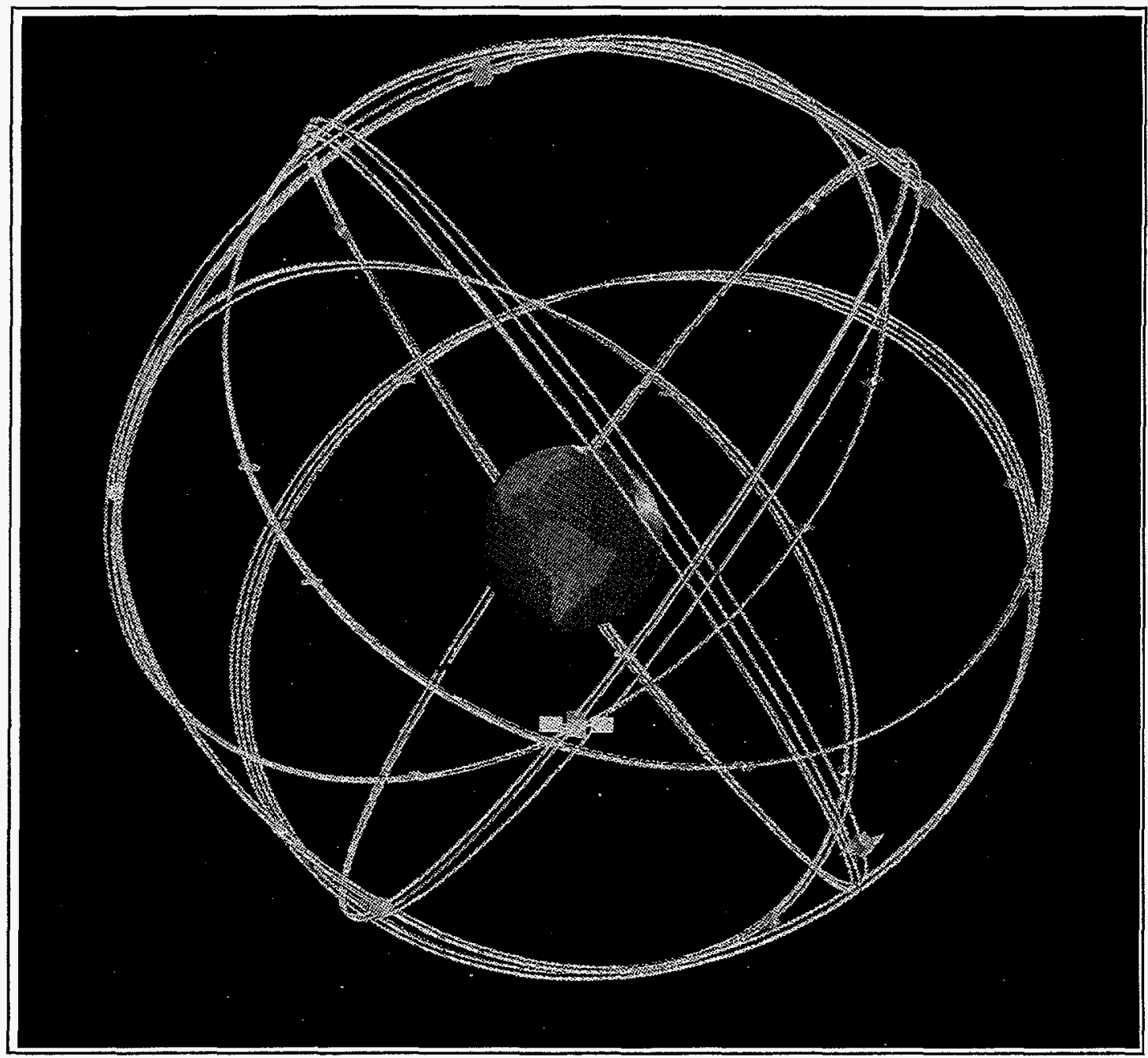

Figure 4. GPS Satellite Orbit \& Path Simulation 
zon of the earth from the spacecraft, that is a plane that cuts through that horizon. If however, some physical phenomena (i.e., sensor sensitivity) prevents visibility to the horizon, this limitation is often quantified by indicating the minimum elevation angle, as measured from the satellite to a plane tangent to the earth. The coverage region for one satellite varies with respect to the elevation angle. Zero degrees is the best case, and greater angles would be less desirable. The simulation is able to show the horizon coverage as the elevation angle varies from 0 to larger degrees.

Another important aspect of coverage is known as solar inhibit. In order to keep the optical sensor onboard the spacecraft from continuously triggering, the sensor is turned off when the sun enters the field of view. The critical portions of the orbit are determined by monitoring the angle formed by straight line connection of the centers of the sun, the vehicle, and the earth, the so called SVE angle. The simulation dynamically computes the SVE for each satellite and effectively turns off the sensor at the appropriate solar inhibit time. During the time that a satellite is inhibited, it is turned red in the simulation. This outage effect comes into play in the computation and display of

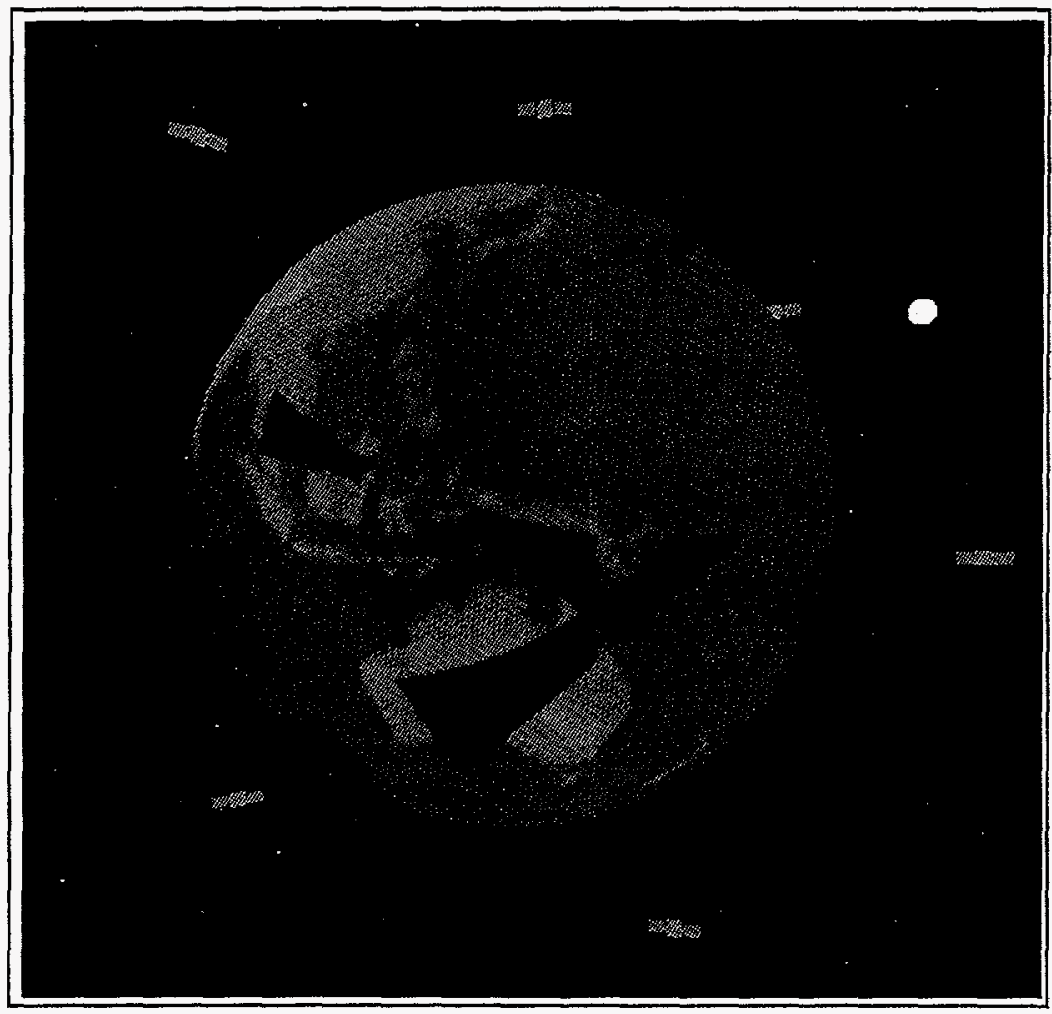

Figure 5. Dynamic Sensor Coverage Map 
sensor coverage maps. The simulation dynamically computes the earth coverage for each satellite and displays the areas of the earth not covered by a selectable minimum number of satellites.

Figure 5. shows one frame of the real-time collective coverage simulation. Areas of the earth without the required coverage (in this case, a minimum of 4 satellites in view) are colored in bright red. With 24 satellites, and assuming an elevation angle of 0 degrees, coverage of the entire earth is normally very good. Areas of non-coverage (i.e., less than 4 satellites in view) occur if we raise the elevation angle, or at certain times when a satellite is solar inhibited, especially if two satellites are simultaneously solar inhibited, or if we require that more than 4 satellites see an area. The virtual environment for this application dynamically and instantaneously shows the sensor coverage from any viewpoint at any time.

\subsection{Terrain and Abstract Data Sets}

As an adjunct to the satellite simulation discussed in 4., virtual environments of terrain data and abstract data sets were explored utilizing once again the VR model discussed in 2 . These types of data sets may be important to display in a satellite application and could potentially be imple-

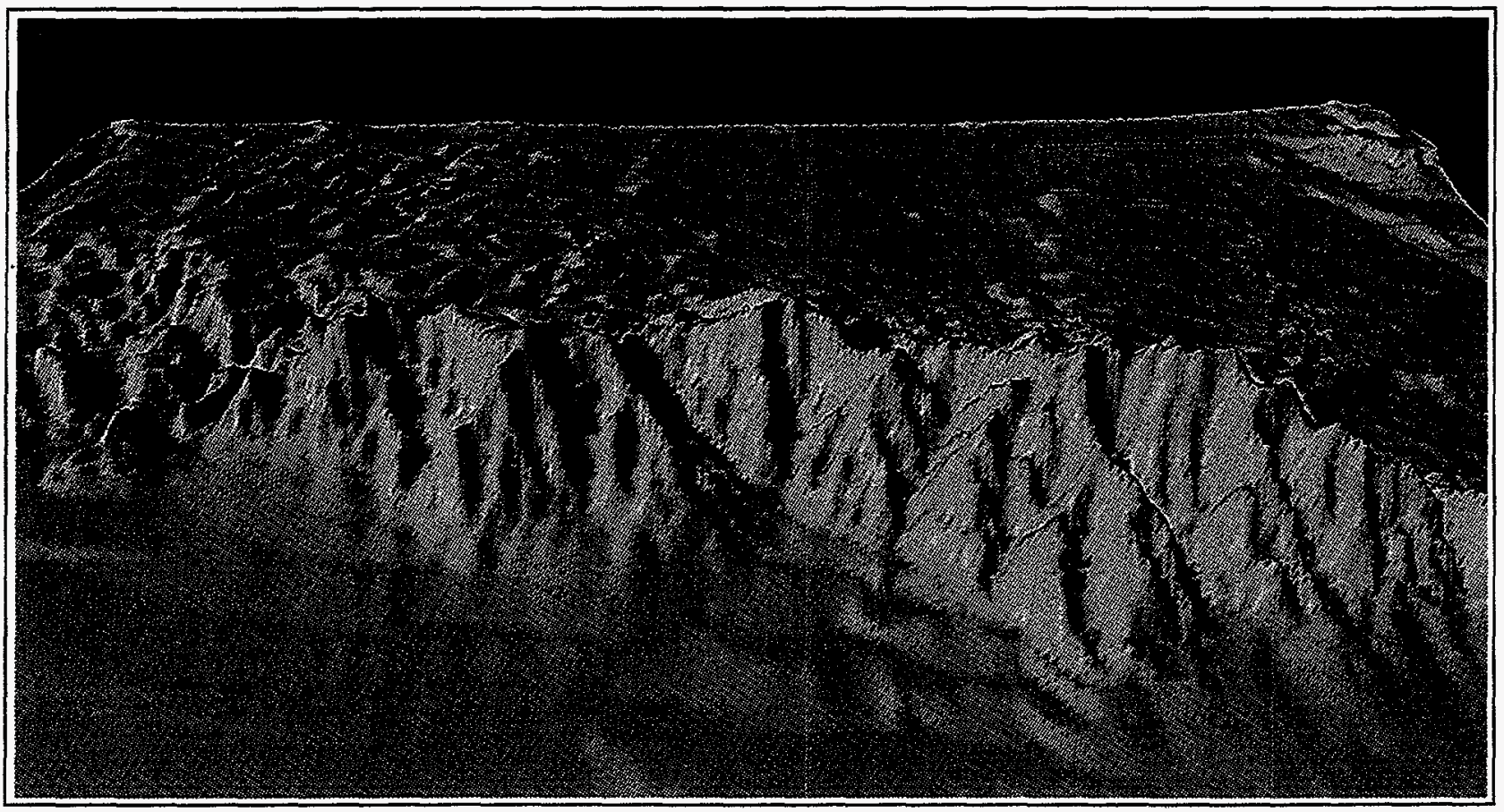

Figure 6. Terrain Elevation Data 
mented as sub-worlds within the main satellite simulation. Figure 6 . is an example of the terrain elevation display.

Abstract data sets are difficult to represent to the human sensory system. Classically this information has only been presented in 2D or at best 3D flat screen graphical displays. An advantage that VR brings to this problem is that it is multi-sensory - both for input sensors and output effectors. Now the application programmer has more options available for presenting this multi-dimensional information to the user. Not only is the "infinite" display of the immersive VR environment available but such things as sound, voice prompting, voice commands, and 3D manipulators become available for understanding and analyzing the data. One example of such an application is shown in Figure 7. This work involved the display of multi-dimensional seismic data. A three dimensional representation of the data is displayed within a virtual environment that may be controlled by the participant through voice commands. The user may move forward or backward through the data set by issuing voice commands. Several devices such as the grid rings shown in figure 7 are controllable also by voice commands and aid the user in analyzing the information.

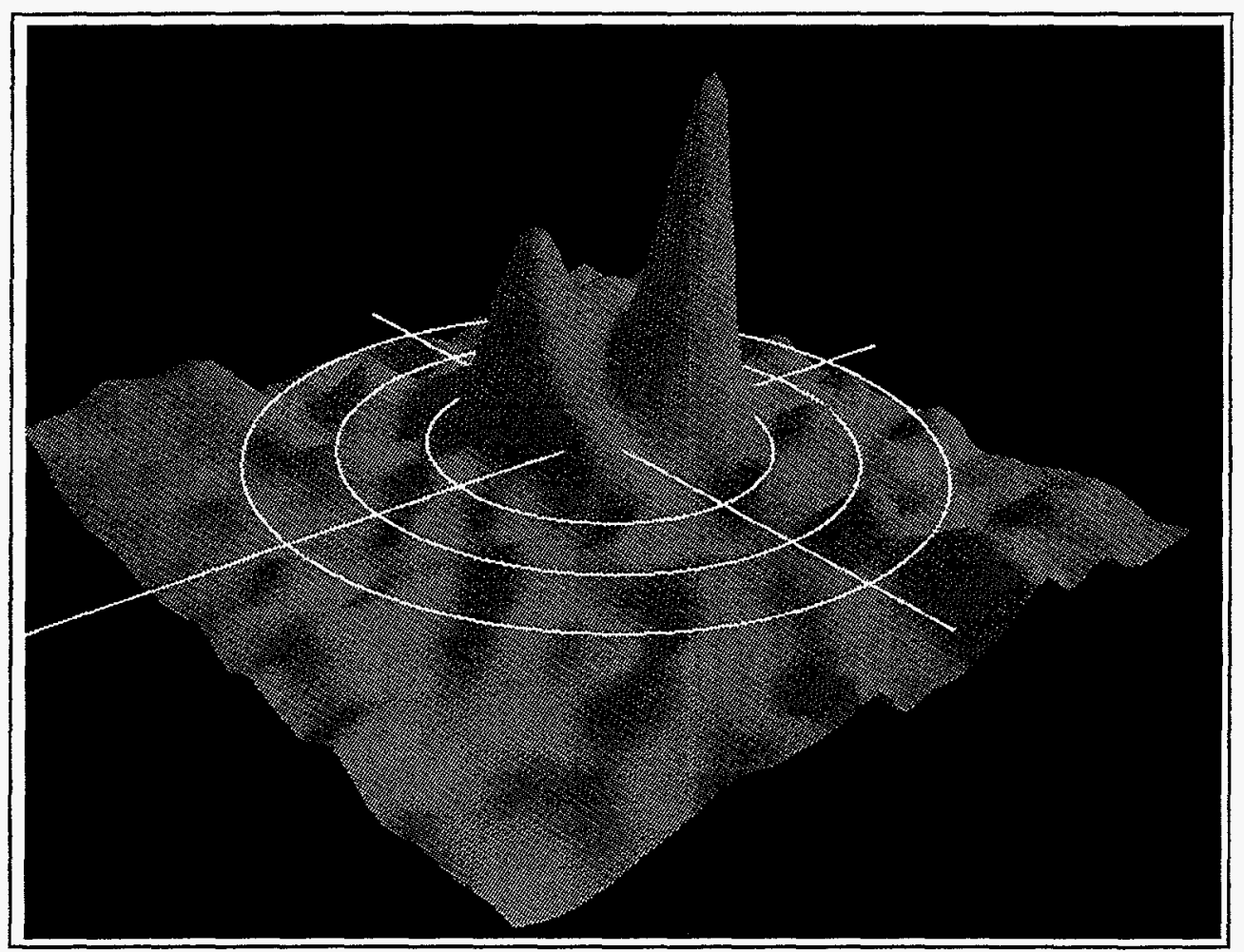

Figure 7. Multi-dimensional Data Visualization 


\subsection{Robotics Application}

Many DOE tasks must be carried out in environments too dangerous for humans. An example is environmental remediation tasks such as removing the waste from leaking underground storage tanks. In order to program a robot to work in such environments, a model of the environment is needed, so that the robot knows where the obstacles and objects of interest are. In the case of environments such as the waste tanks, such models do not exist and must be created prior to robot programming. The purpose of this research was to develop such an interactive model building system using virtual reality and telepresence techniques.

This work included the building of a prototype system which demonstrated the ability to interactively build a graphical model.This prototype system is able to place models (cylinders of known orientation) within 2 inches of their world position and within 0.5 inches of their actual dimensions. The prototype system uses a 3-DOF stereo camera platform slaved to a stereoscopic viewer. The graphical model is also stereo and slaved to the movements of the user's head via the viewer. Methods for registering the graphics and the live video and for interactively building the model were developed. Voice commands and audio feedback were added to aide the user in the modeling task. Computer vision algorithms were developed to extract information about the object of interest. This information was used to build the graphical model.

The major milestones of this work are:

- Integration of a stereo camera system, stereo viewer, and development of a graphical overlay capability.

- Modification of a robotics graphical modeling package to allow socket communication with other devices and software.

- Modeling of the stereo camera platform for calibration purposes.

- Development of a 3D pointer for interaction using a joystick and buttons box.

- Calibration of the stereo CCD cameras on the platform. Camera calibration gives the transformation matrix from world space to image space. Software was written that allows the transformation from image space to world space.

- Development of image processing software to extract edges from the camera platform images.

- Development of a demonstration which illustrates the ability to interactively build models with the cameras fixed.

- Development of software to register graphics and video when the cameras are moving

Details of this work may be found in the publication:

"Interactive Graphical Model Building Using Telepresence and Virtual Reality" C. Cooke and S. Stansfield, submitted to IEEE International conference on Robotics and Automation. 5/8-13 1993. San Diego, CA. 


\subsection{Conclusions}

The virtual reality concept is a very promising step forward in the effort to more effectively use computers for the benefit of mankind. It introduces a new approach in the utilization of computers by taking better advantage of what the computer does best versus what human beings do best. Humans are good at pattern recognition, evaluation, and an overall sense of context. Computers are best at computations, repetitive steps, data storage and retrieval, and remembering. A new division of labor that maximizes these assets cannot help but improve the efficiency of knowledge gained through information processed by our computers. The technology is, even after 10 years of research and development, still infantile in many ways. It is, it seems, a simple concept with many issues and difficulties in implementation. Human psychology and human perception are just a couple of the terribly complex issues that come into play in bridging the gap between concept and realization.

We feel that this research has proven the utility of fully interactive real-time virtual environments and multi-dimensional data visualization in satellite and robotic applications. It also suggests the usefulness of virtual reality in a number of other applications such as training, strategic planning, environmental monitoring, and defense to name just a few. Much more fundamental research is needed on the technology, but at the same time, application "pull" must provide the impetuous for this research and definition of needs that must be satisfied by the technology. Potential users should not be deterred from experimenting with VR on applications because of the fear of some deficiencies of the technology. The concepts are sound and with national focus and funding of further research and with users willing to pioneer the use of the technology, the vision and dreams of the VR innovators will be realized. 


\subsection{REFERENCES}

Kramer, Gregory., "Auditory Display" Sonification, Audification, and Auditory Interfaces. Proceedings Volume XVIII, Santa Fe Institute Studies in the Sciences of Complexity, 1994.

Ellis, Stephen R., "Pictorial communication in virtual and real environments". NASA Ames Research Center and University of California, Berkeley, USA. 1993.

Pimentel, Ken., Teixeira, Kevin., "Virtual Reality - Through the new looking glass". 1993

Durlach, Nathaniel I., Mavor, Anne S., "Virutal Reality - Scientific and Technological Challenges", Committee on Virtual Reality Research and Development Commission on Behavioral and Social Sciences and Education Commission on Physical Sciences, Mathematics, and Applications. National Research Council. 1995.

Satava, Richard M., Morgan, Karen., Sieburg, Hans B., Mattheus, Ruddy., Christensen, Jens P., "Interactive Technology and the New Paradigm for Healthcare". Medicine Meets Virtual Reality III Proceedings, San Diego January 19-22, 1995. 


\section{APPENDIX A - LDRD SUMMARY}

Refereed publications resulting from the work:

"Interactive Graphical Model Building Using Telepresence and Virtual Reality" C. Cooke and S. Stansfield, submitted to IEEE International conference on Robotics and Automation. 5/8-13 1993. San Diego, CA.

All other reports and publications resulting form the work:

"Virtual Reality -- From Research to Real Applications: A Guideline to Applications" G.Q. Van Slambrook, D. P. Holloway, V. P. Holmes, B. A. Malm, May 27, 1993.

Number of patent disclosures:

None.

Number of patent applications:

None.

Number of patents:

None.

Number of Copyrights:

None.

Number of students:

1.

Number of post docs:

None.

Number of permanent technical or scientific staff:

None.

Number of awards:

None.

Number of new non-LDRD funded projects and their amounts:

2.

Qualitative assessment about the completion of final milestones for the project - 95\%.

Qualitative assessment about the direction of the project as a result of research or other findings:

1. Goals met, hypothesis proved. 


\section{Distribution:}

2 MS 1436 Donna L. Chavez, LDRD Office, 4523

21138 Dan Holloway, 6532

41138 Gerald A. Van Slambrook, 6532

19018 Central Technical Files, 8940-2

50899 Technical Library, 4414

20619 Review \& Approval Desk, 12690

For DOE/OSTI 